\title{
NOTE ABOUT SQUARE FUNCTION ESTIMATES AND UNIFORMLY RECTIFIABLE MEASURES
}

\author{
HENRI MARTIKAINEN AND MIHALIS MOURGOGLOU
}

(Communicated by Alexander Iosevich)

\begin{abstract}
We generalise and offer a different proof of a recent $L^{2}$ square function estimate on uniformly rectifiable sets by Hofmann, Mitrea, Mitrea and Morris. The proof is a short argument using the $\alpha$-numbers of Tolsa.
\end{abstract}

\section{INTRODUCTION}

We will deal with certain square function estimates in $\mathbb{R}^{d}$ involving the following class of kernels:

1.1. Definition. Let $\gamma_{1}, \gamma_{2}>0$. We say that $S \in K_{\gamma_{1}, \gamma_{2}}\left(\mathbb{R}^{d}\right)$ if $S: \mathbb{R}^{d} \times \mathbb{R}^{d} \backslash$ $\left\{(x, x): x \in \mathbb{R}^{d}\right\} \rightarrow \mathbb{R}$ satisfies for some $C<\infty$ that

$$
|S(x, y)| \leq \frac{C}{|x-y|^{\gamma_{1}}}
$$

and

$$
\left|S(x, y)-S\left(x, y^{\prime}\right)\right| \leq C \frac{\left|y-y^{\prime}\right|^{\gamma_{2}}}{|x-y|^{\gamma_{1}+\gamma_{2}}}
$$

whenever $\left|y-y^{\prime}\right| \leq|x-y| / 2$. For $\gamma>0$ we set $K_{\gamma}\left(\mathbb{R}^{d}\right)=K_{\gamma, 1}\left(\mathbb{R}^{d}\right)$.

In this paper we denote $A \lesssim B$ if $A \leq C B$ for some absolute constant $C$, and $A \sim B$ if $B \lesssim A \lesssim B$

Suppose $0<n<d$. We say that a Radon measure $\mu$ in $\mathbb{R}^{d}$ is $n$-Ahlfors-David Regular (or $n$-ADR) if $\mu(B(x, r)) \sim r^{n}$ for all $x \in \operatorname{spt} \mu$ and $0<r \leq \operatorname{diam}(\operatorname{spt} \mu)$. Consider a kernel $S \in K_{n+\beta, \gamma}\left(\mathbb{R}^{d}\right)$ for some $\beta, \gamma>0$. Given $f \in L^{2}(\mu) \cup L^{\infty}(\mu)$ and $x \in \mathbb{R}^{d} \backslash$ spt $\mu$ we define the absolutely convergent integral

$$
T_{S, \mu} f(x)=\int S(x, y) f(y) d \mu(y) .
$$

In what follows we denote $E=\operatorname{spt} \mu$. For minor convenience, we shall always assume $\operatorname{diam}(E)=\infty$. We are interested in the square function estimate

$$
\int_{\mathbb{R}^{d} \backslash E}\left|T_{S, \mu} f(x)\right|^{2} \operatorname{dist}(x, E)^{2 \beta-(d-n)} d x \lesssim \int_{E}|f(y)|^{2} d \mu(y), \quad f \in L^{2}(\mu) .
$$

Received by the editors January 6, 2015 and, in revised form, October 2, 2015.

2010 Mathematics Subject Classification. Primary 42B20.

Key words and phrases. Square function, $\alpha$-numbers, uniform rectifiability.

The first author was supported by the Academy of Finland through the grant Multiparameter dyadic harmonic analysis and probabilistic methods, and is a member of the Finnish Centre of Excellence in Analysis and Dynamics Research.

The research of the second author was supported by the ERC grant 320501 of the European Research Council (FP7/2007-2013). 
David and Semmes [5], and more recently Hofmann, Mitrea, Mitrea and Morris [8] have studied the square function estimate (1.2) in the context of uniformly rectifiable sets in co-dimension one (i.e. $d=n+1$ ).

1.3. Definition. Let $n$ be an integer. A measure $\mu$ in $\mathbb{R}^{d}$ is $n$-UR (uniformly rectifiable) if it is $n$-ADR and it satisfies the big pieces of Lipschitz images (BPLI) property. This means that there exist $\theta, M>0$ such that for all $x \in E=\operatorname{spt} \mu$ and $r>0$ we have a Lipschitz mapping $g$ from the ball $B_{n}(0, r) \subset \mathbb{R}^{n}$ to $\mathbb{R}^{d}$ with $\operatorname{Lip}(g) \leq M$ and

$$
\mu\left(B(x, r) \cap g\left(B_{n}(0, r)\right)\right) \geq \theta r^{n} .
$$

A closed set $E \subset \mathbb{R}^{d}$ is $n$-ADR if $\mu=\left.\mathcal{H}^{n}\right|_{E}$ is $n$-ADR, and it is called uniformly $n$-rectifiable if, further, $\left.\mathcal{H}^{n}\right|_{E}$ is uniformly $n$-rectifiable. Here $\mathcal{H}^{n}$ denotes the $n$ dimensional Hausdorff measure in $\mathbb{R}^{d}$.

Hofmann, Mitrea, Mitrea and Morris [8, Corollary 5.7] proved that the square function estimate (1.2) holds, if $\mu=\left.\mathcal{H}^{n}\right|_{E}$ for a given $n$-UR set $E \subset \mathbb{R}^{n+1}$ and $S(x, y)=\left(\partial_{j} K\right)(x-y)$ for some fixed $j \in\{1, \ldots, n+1\}$, where $K \in C^{2}\left(\mathbb{R}^{n+1} \backslash\{0\}\right)$, $K$ is odd and $K(\lambda x)=\lambda^{-n} K(x)$ for $\lambda>0, x \in \mathbb{R}^{n+1} \backslash\{0\}$. Such kernels $S$ are kernels of convolution form in $K_{n+1}\left(\mathbb{R}^{n+1}\right)$ and satisfy

$$
T_{S, L} 1(x):=\int_{L} S(x, y) d \mathcal{H}^{n}(y)=0
$$

for every $n$-plane $L$ and $x \notin L$. Much earlier, David and Semmes [5] had proved the case where $K(x)=x_{i} /|x|^{n+1}$ for some $i \in\{1, \ldots, n+1\}$. The proof in 8 ] is relatively complicated having the following steps:

(1) Using a general local $T b$ theorem one proves that having big pieces of square function estimates (BPSFE) is enough for (1.2). See Definition 4.1 of [8] for the definition of BPSFE.

(2) One proves (1.2) in the case that $S(x, y)=\left(\partial_{j} K\right)(x-y)$ like above and $E$ is a Lipschitz graph. This uses, among other things, Fourier analysis and borrows some techniques from the earlier papers [2], 6], 7] and [9].

(3) One then considers a set $E$ which has big pieces of Lipschitz graphs (BPLG), or rather $(\mathrm{BP})^{k} \mathrm{LG}$ for some $k$ (big pieces of big pieces of...). Then (1.2) follows (for $S$ like above) from the Lipschitz graph case using the theorem about the sufficiency of BPSFE. See Definition 5.1 of 8 for the definitions of BPLG and $(\mathrm{BP})^{k} \mathrm{LG}$.

(4) Finally, one uses a deep geometric fact by Azzam and Schul [1] which says that a UR set $E$ has $(\mathrm{BP})^{2} \mathrm{LG}$.

Our aim is to provide a new and much simpler proof of the above result, and also to prove a more general result.

We now discuss the $T 1$ theorem in our setting. It is an important tool in our proof, since it is extremely useful for verifying the square function estimate (1.2). It works assuming only that the measure $\mu$ is $n$-ADR. Before stating it, we need to introduce some dyadic notation. Let $\mathcal{D}(E)$ be a dyadic structure in $E=\operatorname{spt} \mu$ (that is, a collection of David or Christ cubes). We refer to 3 for the construction of dyadic systems in metric spaces. This means that $\mathcal{D}(E)=\bigcup_{j} \mathcal{D}_{j}(E)$, and each cube (this is just terminology) $Q \in \mathcal{D}_{j}(E)$ satisfies $Q \subset E, c^{-1} 2^{-j} \leq \operatorname{diam}(Q) \leq 2^{-j}$ and $\mu(Q) \sim 2^{-j n}$. For $Q \in \mathcal{D}_{j}$ we set $\ell(Q)=2^{-j}$. These sets enjoy the usual structural properties of dyadic cubes i.e. for two cubes $Q, R \in \mathcal{D}(E)$ either $Q \cap R=\emptyset$ or one 
of them is contained in the other. For a dyadic cube $R \in \mathcal{D}(E), R^{(k)}$ denotes the unique cube $S \in \mathcal{D}(E)$ such that $R \subset S$ and $\ell(S)=2^{k} \ell(R)$.

For a true cube $W \subset \mathbb{R}^{d}$ we denote its side length also by $\ell(W)$. Let $\mathcal{W}$ denote the collection of maximal cubes $W$ from the standard dyadic grid of $\mathbb{R}^{d}$ for which there holds that $3 W \subset \mathbb{R}^{d} \backslash E$. Then we have that $\operatorname{dist}(x, E) \sim \ell(W)$ for every $x \in 2 W$. To each $W \in \mathcal{W}$ we associate precisely one $Q(W) \in \mathcal{D}(E)$ for which $\operatorname{dist}(Q(W), W) \sim \ell(W) \sim \ell(Q(W))$. For every $Q \in \mathcal{D}(E)$ we then define the Whitney region associated to $Q$ by setting

$$
W_{Q}=\bigcup\{W \in \mathcal{W}: Q(W)=Q\} .
$$

The Carleson box $\widehat{R}$ is defined by

$$
\widehat{R}=\bigcup_{\substack{Q \in \mathcal{D}(E) \\ Q \subset R}} W_{Q} .
$$

The above is a way to produce Whitney regions which works in this generality. The exact way of producing them is not of great importance. Rather, it is the properties that they enjoy which we shall now list. We have that the sets $W_{Q}$, $Q \in \mathcal{D}(E)$, are disjoint, $\mathbb{R}^{d} \backslash E=\bigcup_{W \in \mathcal{W}} W=\bigcup_{Q \in \mathcal{D}(E)} W_{Q}, \operatorname{dist}(x, E) \sim \ell(Q)$ if $x \in W_{Q}$, and $\left|W_{Q}\right| \sim \ell(Q)^{d}$ (for $W_{Q} \neq \emptyset$ ).

We are ready to state the $T 1$ theorem from [8, Theorem 3.2]. It says that the square function estimate (1.2) is equivalent to

$$
\sup _{R \in \mathcal{D}(E)} \frac{1}{\mu(R)} \int_{\widehat{R}}\left|T_{S, \mu} 1(x)\right|^{2} \operatorname{dist}(x, E)^{2 \beta-(d-n)} d x<\infty .
$$

We now formulate our theorem about square function estimates for UR measures.

1.6. Theorem. Let $n, d$ be integers and $0<n<d$. Suppose $S$ is a kernel which satisfies $S \in K_{n+\beta}\left(\mathbb{R}^{d}\right)$ for some $\beta>0$. Let $\mu$ be an $n$-UR measure in $\mathbb{R}^{d}$ with $E=\operatorname{spt} \mu$. If the Carleson condition

$$
\sup _{R \in \mathcal{D}(E)} \frac{1}{\ell(R)^{n}} \int_{\widehat{R} L: \operatorname{dist}(x, L) \sim \operatorname{dist}(x, E)}\left|T_{S, L} 1(x)\right|^{2} \operatorname{dist}(x, E)^{2 \beta-(d-n)} d x<\infty
$$

holds, then the square function estimate (1.2) holds. Here, for a given $x$, the supremum is taken over all the $n$-planes $L \subset \mathbb{R}^{d}$ for which $\operatorname{dist}(x, L) \sim \operatorname{dist}(x, E)$. The notation $T_{S, L}$ is defined in (1.4).

The $T 1$ theorem is extremely useful for verifying the square function estimate (1.2). However, the object

$$
T_{S, \mu} 1(x)=\int_{E} S(x, y) d \mu(y)
$$

might not be so easy to get a hold of if $E$ is not something "geometrically simple". In the case that $\mu$ is not only $n$-ADR but also $n$-UR and $S \in K_{n+\beta}\left(\mathbb{R}^{d}\right)$ (= $K_{n+\beta, 1}\left(\mathbb{R}^{d}\right)$ ), the Carleson type condition (1.7), where one only needs to integrate over $n$-planes, turns out to be sufficient for (1.2). Such a condition can be preferable when $S$ is seen to have some special cancellation on $n$-planes $L$ (for example, when $T_{S, L} 1$ vanishes).

Theorem 1.6 is stated in all co-dimensions, but it is at least interesting in the case of co-dimension one (meaning that $d=n+1$ ). Indeed, it generalises Corollary 5.7 
of [8], which we discussed above. Recall that $T_{S, L} 1$ vanishes for their kernels. The proof of Theorem 1.6 is completely different from the proof of the above-referenced result in [8]. We work directly with the given UR measure $\mu$ making no reductions. Inspired by 4 we use the technology of $\alpha$-numbers without resorting to Fourier analysis. In particular, we don't have to restrict to convolution form kernels. We note that [8] does also include some results about variable coefficient kernels for which they need their convolution form theorem combined with some spherical harmonics extensions.

We will now introduce the $\alpha$-numbers, which are needed for our proof. The big pieces of Lipschitz images property stated in Definition 1.3 seems to be the preferred definition of uniform rectifiability. However, it is equivalent to a huge plethora of different conditions. For us the crucial one is the one using the so called $\alpha$-numbers of Tolsa [10]. For two Borel measures $\sigma$ and $\nu$ in $\mathbb{R}^{d}$ and a closed ball $B \subset \mathbb{R}^{d}$ we set

$$
\operatorname{dist}_{B}(\sigma, \nu)=\sup \left\{\left|\int f d \sigma-\int f d \nu\right|: \operatorname{Lip}(f) \leq 1, \operatorname{spt} f \subset B\right\} .
$$

We fix $M \sim 1$ so that given $Q \in \mathcal{D}(E)$ we have $2 W \subset B\left(c_{Q}, M \ell(Q)\right)$ if $Q(W)=Q$. Here $c_{Q}$ denotes the centre of $Q$ - it is a point in $Q$ such that $d\left(c_{Q}, E \backslash Q\right) \gtrsim \ell(Q)$. To each cube $Q \in \mathcal{D}(E)$ we also associate the ball $B_{Q}=B\left(c_{Q}, 2 M \ell(Q)\right)$. For $Q \in \mathcal{D}(E)$, we define

$$
\alpha(Q)=\frac{1}{\ell(Q)^{n+1}} \inf _{c \geq 0, L} \operatorname{dist}_{B_{Q}}\left(\mu,\left.c \mathcal{H}^{n}\right|_{L}\right),
$$

where the infimum is taken over all the constants $c \geq 0$ and all the $n$-planes $L$ for which $L \cap \frac{1}{2} B_{Q} \neq \emptyset$. The constant $\alpha(Q)$ measures in a scale invariant way how close $\mu$ is to a flat $n$-dimensional measure in the ball $B_{Q}$. We also choose $c_{Q}$ and $L_{Q}$ which minimise $\alpha(Q)$. We always have that $c_{Q} \lesssim 1$, and that if $\alpha(Q)$ is small enough, then also $c_{Q} \gtrsim 1$ (see [10]). The key result of [10] for us is that if $\mu$ is $n$-UR, then for all $R \in \mathcal{D}(E)$ we have that

$$
\sum_{\substack{Q \in \mathcal{D}(E) \\ Q \subset R}} \alpha(Q)^{2} \mu(Q) \lesssim \mu(R) .
$$

1.9. Remark. Notice that if $S \in K_{n+\beta}, \beta>0$, has the cancellation property $T_{S, L} 1 \equiv$ 0 for every $n$-plane, then (1.2) holds for every $n$-UR measure $\mu$. Our Carleson condition (1.7) is a formal relaxation of this. The absolutely most naive relaxation would be to assume that

$$
\int_{\mathbb{R}^{d} \backslash L}\left|T_{S, L} f(x)\right|^{2} \operatorname{dist}(x, L)^{2 \beta-(d-n)} d x \leq C \int_{L}|f(y)|^{2} d \mathcal{H}^{n}(y), \quad f \in L^{2}(L),
$$

for every $n$-plane $L$ and some constant $C<\infty$ independent of $L$. But such a property does not imply much: the square function estimate can then fail on a sphere even for a positive kernel $S$. For example, define

$$
S(x, y):=\frac{1_{B_{1}}(x)}{H(x)+|x-y|^{2}}, \quad x, y \in \mathbb{R}^{2}, x \neq y .
$$

Here $B_{1}:=B(0,1)$ and $H: \mathbb{R}^{2} \rightarrow[0, \infty], H(x):=h(\operatorname{dist}(x, O))$, where $O=\partial B_{1}$,

$$
h(t):=t^{2}\left(1+\log ^{+} \frac{1}{t}\right),
$$


and $\log ^{+} t=\max \{\log t, 0\}$ for $t>0$. This is a minor side note, which is still somewhat tedious to check. T. Orponen provided us with this example and the calculations verifying it. We give only minor hints of the details.

To check the failure of the square function estimate on the sphere notice that

$$
T_{S, O} 1(x) \gtrsim H(x)^{-1 / 2}
$$

for $99 / 100<|x|<1$. Therefore, we have that

$$
\begin{aligned}
\int_{\mathbb{R}^{2} \backslash O}\left|T_{S, O} 1(x)\right|^{2} \operatorname{dist}(x, O) d x & \gtrsim \int_{99 / 100<|x|<1} \frac{d x}{\operatorname{dist}(x, O)(1+\log (1 / \operatorname{dist}(x, O)))} \\
& \sim \int_{0}^{1 / 100} \frac{d r}{r(1+\log (1 / r))}=\infty
\end{aligned}
$$

proving that the square function estimate does not hold for the UR set $O$. But we can show that it does hold uniformly on every line $L$.

The verification of this is more involved. Fix a line $L$, and an arbitrary dyadic interval $I_{0} \subset L$. By the $T 1$ theorem it is enough to verify the estimate

$$
\int_{\widehat{I}_{0}}\left|T_{S, L} 1(x)\right|^{2} \operatorname{dist}(x, L) d x \lesssim \ell\left(I_{0}\right) .
$$

We rewrite and estimate the left hand side as follows:

$$
\begin{aligned}
\int_{\widehat{I}_{0}}\left|T_{S, L} 1(x)\right|^{2} \operatorname{dist}(x, L) d x & \sim \sum_{I \subset I_{0}} \ell(I) \int_{W_{I}}\left|T_{S, L} 1(x)\right|^{2} d x \\
& \lesssim \sum_{I \subset I_{0}} \ell(I) \int_{W_{I} \cap B_{1}} \min \left(\frac{1}{H(x)}, \frac{1}{\ell(I)^{2}}\right) d x .
\end{aligned}
$$

Further estimation of this is somewhat involved (we need to handle separately long and small intervals $I$ ). We omit the details.

\section{Proof of the square function estimate}

In this section we give a proof of the square function estimate (1.2) under the UR hypothesis and (1.7). The proof is short so we are quite generous with the details.

Proof of Theorem 1.6. We will verify the $T 1$ condition (1.5). To this end, fix $R \in$ $\mathcal{D}(E)$. Recalling the definition of $\widehat{R}$ we need to prove that

$$
\operatorname{Car}(R):=\sum_{\substack{Q \in \mathcal{D}(E) \\ Q \subset R}} \ell(Q)^{2 \beta-(d-n)} \int_{W_{Q}}\left|T_{S, \mu} 1(x)\right|^{2} d x \lesssim \mu(R) .
$$

For every $Q \in \mathcal{D}(E)$ and $x \in W_{Q}$ we want to prove that

$$
\begin{aligned}
\left|T_{S, \mu} 1(x)\right| & \lesssim \frac{1}{\ell(Q)^{\beta}} \sum_{\substack{P \in \mathcal{D}(E) \\
P \supset Q}}\left(\frac{\ell(Q)}{\ell(P)}\right)^{\beta} \alpha(P)+\sup _{L: \operatorname{dist}(x, L) \sim \operatorname{dist}(x, E)}\left|T_{S, L} 1(x)\right| \\
& =: U_{1}(Q)+U_{2}(x) .
\end{aligned}
$$


Indeed, notice that

$$
\begin{aligned}
\sum_{\substack{Q \in \mathcal{D}(E) \\
Q \subset R}} \ell(Q)^{2 \beta-(d-n)} \int_{W_{Q}} U_{1}(Q)^{2} d x & \lesssim \sum_{Q: Q \subset R} \mu(Q) \sum_{P: Q \subset P \subset R}\left(\frac{\ell(Q)}{\ell(P)}\right)^{\beta} \alpha(P)^{2} \\
& +\sum_{Q: Q \subset R} \mu(Q) \sum_{P: R \subset P}\left(\frac{\ell(Q)}{\ell(P)}\right)^{\beta} \alpha(P)^{2}=I_{1}+I_{2} .
\end{aligned}
$$

Using the Carleson property of the $\alpha$-numbers (1.8) we see that

$$
I_{1}=\sum_{P: P \subset R} \alpha(P)^{2} \sum_{Q: Q \subset P}\left(\frac{\ell(Q)}{\ell(P)}\right)^{\beta} \mu(Q) \lesssim \sum_{P: P \subset R} \alpha(P)^{2} \mu(P) \lesssim \mu(R) .
$$

The term $I_{2}$ is completely elementary (we just estimate $\alpha(P) \lesssim 1$ and do not need the Carleson property of the $\alpha$-numbers):

$$
I_{2} \lesssim \sum_{Q: Q \subset R} \mu(Q)\left(\frac{\ell(Q)}{\ell(R)}\right)^{\beta} \sum_{P: R \subset P}\left(\frac{\ell(R)}{\ell(P)}\right)^{\beta} \lesssim \sum_{Q: Q \subset R} \mu(Q)\left(\frac{\ell(Q)}{\ell(R)}\right)^{\beta} \lesssim \mu(R) .
$$

Next, notice that using (1.7) we have that

$$
\begin{aligned}
& \sum_{\substack{Q \in \mathcal{D}(E) \\
Q \subset R}} \ell(Q)^{2 \beta-(d-n)} \int_{W_{Q}} U_{2}(x)^{2} d x \\
& \quad \sim \int_{\widehat{R} L: \operatorname{dist}(x, L) \sim \operatorname{dist}(x, E)}\left|T_{S, L} 1(x)\right|^{2} \operatorname{dist}(x, E)^{2 \beta-(d-n)} d x \lesssim \ell(R)^{n} \sim \mu(R) .
\end{aligned}
$$

Combining the estimates, we have that (2.1) implies that $\operatorname{Car}(R) \lesssim \mu(R)$. So it only remains to prove (2.1).

Fix $Q \in \mathcal{D}(E)$ and $x \in W_{Q}$. If $\alpha(Q) \geq c_{0}$ we have the trivial estimate

$$
\left|T_{S, \mu} 1(x)\right| \lesssim \operatorname{dist}(x, E)^{-\beta} \sim \ell(Q)^{-\beta} \lesssim \ell(Q)^{-\beta} \alpha(Q) \leq \frac{1}{\ell(Q)^{\beta}} \sum_{P: P \supseteq Q}\left(\frac{\ell(Q)}{\ell(P)}\right)^{\beta} \alpha(P) .
$$

Therefore, we may assume that $\alpha(Q)<c_{0}$ for a small parameter $c_{0}$ to be chosen. We will use this to show that $B\left(x, c_{1} \ell(Q)\right) \cap L_{Q}=\emptyset$ (here $c_{1}>0$ is a small enough dimensional constant). To this end, let us first show that

$$
\sup _{y \in L_{Q} \cap B\left(c_{Q}, M \ell(Q)\right)} \frac{\operatorname{dist}(y, E)}{M \ell(Q)} \leq C c_{0}^{1 /(n+1)}=\epsilon_{0} .
$$

Suppose $y \in B\left(c_{Q}, M \ell(Q)\right) \cap L_{Q}$ and set $\tau=\tau_{y}=\operatorname{dist}(y, E) / M \ell(Q)$. Notice here that $\tau \in[0,1]$ and $B(y, \tau M \ell(Q)) \subset \mathbb{R}^{d} \backslash E$. Let $\phi$ satisfy $1_{B(y, \tau M \ell(Q) / 2)} \leq \phi \leq$ $1_{B(y, \tau M \ell(Q))}$ and $\operatorname{Lip}(\phi) \sim(\tau \ell(Q))^{-1}$. Note that $\operatorname{spt} \phi \subset B\left(c_{Q}, 2 M \ell(Q)\right)=B_{Q}$ and spt $\phi \subset \mathbb{R}^{d} \backslash E$. Therefore, we have that

$$
\tau^{-1} c_{0} \ell(Q)^{n} \gtrsim \operatorname{Lip}(\phi) \alpha(Q) \ell(Q)^{n+1} \geq\left. c_{Q} \int \phi d \mathcal{H}^{n}\right|_{L_{Q}} \gtrsim_{M} \tau^{n} \ell(Q)^{n} .
$$

Here we used that $c_{Q} \gtrsim 1$ since $\alpha(Q)$ is small. This establishes (2.2).

Suppose then that $B\left(x, c_{1} \ell(Q)\right) \cap L_{Q} \neq \emptyset$. Then there exists $W \in \mathcal{W}$ so that $Q(W)=Q$ and there exists $y \in 2 W \cap L_{Q} \subset B\left(c_{Q}, M \ell(Q)\right) \cap L_{Q}$ (the constant $c_{1}$ is so small that $B\left(x, c_{1} \ell(Q)\right) \subset 2 W$ if $\left.x \in W\right)$. But, in view of (2.2), this means that

$$
\ell(W) \sim \operatorname{dist}(y, E) \leq \epsilon_{0} M \ell(Q) \sim \epsilon_{0} \ell(W),
$$


which is a contradiction for a small enough $\epsilon_{0}$ i.e. for a small enough $c_{0}$. We thus conclude that $B\left(x, c_{1} \ell(Q)\right) \cap L_{Q}=\emptyset$.

Recalling that $c_{Q} \lesssim 1$ we estimate

$$
\left|T_{S, \mu} 1(x)\right| \lesssim\left|\int S(x, y) d\left(\mu-\left.c_{Q} \mathcal{H}^{n}\right|_{L_{Q}}\right)(y)\right|+\left|\int S(x, y) d \mathcal{H}^{n}\right|_{L_{Q}}(y) \mid=P_{1}+P_{2} .
$$

We first deal with $P_{1}$. To this end, notice that

$$
P_{1} \leq \sum_{k \geq 1}\left|\int \gamma_{k}(y) S(x, y) d\left(\mu-\left.c_{Q} \mathcal{H}^{n}\right|_{L_{Q}}\right)(y)\right|,
$$

where $\sum_{k>0} \gamma_{k}=1, \gamma_{k}$ is smooth, supported on those $y$ for which $|x-y| \sim 2^{k} \ell(Q)$ and satisfies $\left\|\nabla \gamma_{k}\right\|_{\infty} \lesssim\left(2^{k} \ell(Q)\right)^{-1}$. The key thing is that the corresponding function $\gamma_{0}$ is not needed, since it is supported on $B\left(x, c_{1} \ell(Q)\right)$ and this does not intersect the support of the measure i.e. $E \cup L_{Q}$. We further estimate

$$
\begin{aligned}
P_{1} \leq \sum_{k \geq 1}\left|\int \gamma_{k}(y) S(x, y) d\left(\mu-\left.c_{Q^{\left(k+s_{0}\right)}} \mathcal{H}^{n}\right|_{L_{Q^{\left(k+s_{0}\right)}}}\right)(y)\right| \\
\quad+\sum_{k \geq 1}\left|\int \gamma_{k}(y) S(x, y) d\left(\left.c_{Q^{\left(k+s_{0}\right)}} \mathcal{H}^{n}\right|_{L_{Q^{\left(k+s_{0}\right)}}}-\left.c_{Q} \mathcal{H}^{n}\right|_{L_{Q}}\right)(y)\right|=J_{1}+J_{2}
\end{aligned}
$$

for some $s_{0} \sim 1$ such that spt $\gamma_{k} \subset B_{Q^{\left(k+s_{0}\right)}}$.

The function $y \mapsto \gamma_{k}(y) S(x, y)$ is Lipschitz with

$$
\operatorname{Lip}\left(\gamma_{k}(\cdot) S(x, \cdot)\right) \lesssim\left(2^{k} \ell(Q)\right)^{-n-\beta-1} .
$$

This follows easily by using the size, Lipschitz and support properties of the involved functions. Therefore, we have that

$$
J_{1} \lesssim \sum_{k \geq 1} \alpha\left(Q^{\left(k+s_{0}\right)}\right) \ell\left(Q^{\left(k+s_{0}\right)}\right)^{n+1}\left(2^{k} \ell(Q)\right)^{-n-\beta-1} \lesssim \frac{1}{\ell(Q)^{\beta}} \sum_{P: P \supset Q}\left(\frac{\ell(Q)}{\ell(P)}\right)^{\beta} \alpha(P) .
$$

Let us then estimate $J_{2}$. Let $f_{k}(y):=\left(2^{k} \ell(Q)\right)^{n+1+\beta} \gamma_{k}(y) S(x, y)$ so that $\operatorname{Lip}\left(f_{k}\right)$ $\lesssim 1$ and

$$
J_{2} \leq \sum_{k \geq 1}\left(2^{k} \ell(Q)\right)^{-n-1-\beta} \sum_{j=1}^{k+s_{0}}\left|\int f_{k}(y) d\left(\left.c_{Q^{(j)}} \mathcal{H}^{n}\right|_{L_{Q^{(j)}}}-\left.c_{Q^{(j-1)}} \mathcal{H}^{n}\right|_{L_{Q^{(j-1)}}}\right)(y)\right| .
$$

For a fixed $k$ and $j$ we estimate

$$
\begin{aligned}
\mid \int f_{k}(y) & d\left(\left.c_{Q^{(j)}} \mathcal{H}^{n}\right|_{L_{Q^{(j)}}}-\left.c_{Q^{(j-1)}} \mathcal{H}^{n}\right|_{L_{Q^{(j-1)}}}\right)(y) \mid \\
& \leq c_{Q^{(j)}}\left|\int f_{k}(y) d\left(\left.\mathcal{H}^{n}\right|_{L_{Q^{(j)}}}-\left.\mathcal{H}^{n}\right|_{L_{Q^{(j-1)}}}\right)(y)\right| \\
& +\left|c_{Q^{(j)}}-c_{Q^{(j-1)}}\right|\left|\int f_{k}(y) d \mathcal{H}^{n}\right|_{L_{Q^{(j-1)}}}(y) \mid=K_{1}+K_{2} .
\end{aligned}
$$

To estimate $K_{1}$ we use that $c_{Q^{(j)}} \lesssim 1$ and that

$$
d_{H}\left(L_{Q^{(j-1)}} \cap B_{Q^{\left(k+s_{0}\right)}}, L_{Q^{(j)}} \cap B_{Q^{\left(k+s_{0}\right)}}\right) \lesssim \alpha\left(Q^{(j)}\right) \ell\left(Q^{\left(k+s_{0}\right)}\right) .
$$

Here $d_{H}$ stands for the Hausdorff distance and we have used Lemma 3.4 of [10]. Using this one sees that

$$
K_{1} \lesssim \alpha\left(Q^{(j)}\right)\left(2^{k} \ell(Q)\right)^{n+1}
$$


Lemma 3.4 of [10] also gives that $\left|c_{Q^{(j)}}-c_{Q^{(j-1)}}\right| \lesssim \alpha\left(Q^{(j)}\right)$. After this it is clear that also

$$
K_{2} \lesssim \alpha\left(Q^{(j)}\right)\left(2^{k} \ell(Q)\right)^{n+1}
$$

We conclude that

$$
\begin{aligned}
J_{2} & \lesssim \sum_{k \geq 1}\left(2^{k} \ell(Q)\right)^{-\beta} \sum_{j=1}^{k+s_{0}} \alpha\left(Q^{(j)}\right) \\
& \lesssim \sum_{R: R \supset Q} \ell(R)^{-\beta} \sum_{P: Q \subset P \subset R} \alpha(P) \\
& =\sum_{P: P \supset Q} \alpha(P) \ell(P)^{-\beta} \sum_{R: R \supset P}\left(\frac{\ell(P)}{\ell(R)}\right)^{\beta} \\
& \lesssim \sum_{P: P \supset Q} \alpha(P) \ell(P)^{-\beta}=\frac{1}{\ell(Q)^{\beta}} \sum_{P: P \supset Q}\left(\frac{\ell(Q)}{\ell(P)}\right)^{\beta} \alpha(P) .
\end{aligned}
$$

Combining the estimates for $J_{1}$ and $J_{2}$ we have that

$$
P_{1} \lesssim \frac{1}{\ell(Q)^{\beta}} \sum_{P: P \supset Q}\left(\frac{\ell(Q)}{\ell(P)}\right)^{\beta} \alpha(P) .
$$

For $P_{2}$ notice that we have that $\operatorname{dist}\left(x, L_{Q}\right) \sim \ell(Q) \sim \operatorname{dist}(x, E)$. Therefore, we have that

$$
P_{2} \lesssim \sup _{L: \operatorname{dist}(x, L) \sim \operatorname{dist}(x, E)}\left|T_{S, L} 1(x)\right| .
$$

Combining the estimates for $P_{1}$ and $P_{2}$ we have that (2.1) holds. Therefore, we are done.

\section{ACKNOWLEDGEMENTS}

The authors thank J. Azzam and T. Orponen for discussions related to square functions and UR measures. T. Orponen constructed and verified the details in Remark 1.9 .

\section{REFERENCES}

[1] Jonas Azzam and Raanan Schul, Hard Sard: quantitative implicit function and extension theorems for Lipschitz maps, Geom. Funct. Anal. 22 (2012), no. 5, 1062-1123, DOI 10.1007/s00039-012-0189-0. MR/2989430

[2] Michael Christ, Lectures on singular integral operators, CBMS Regional Conference Series in Mathematics, vol. 77, Published for the Conference Board of the Mathematical Sciences, Washington, DC; by the American Mathematical Society, Providence, RI, 1990. MR.1104656 (92f:42021)

[3] Michael Christ, A T(b) theorem with remarks on analytic capacity and the Cauchy integral, Colloq. Math. 60/61 (1990), no. 2, 601-628. MR1096400 (92k:42020)

[4] Vasilis Chousionis, John Garnett, Triet Le, and Xavier Tolsa, Square functions and uniform rectifiability, Trans. Amer. Math. Soc., to appear, available at http://arxiv.org/abs/1401. 3382.

[5] Guy David and Stephen Semmes, Analysis of and on uniformly rectifiable sets, Mathematical Surveys and Monographs, vol. 38, American Mathematical Society, Providence, RI, 1993. MR.1251061 (94i:28003)

[6] Steve Hofmann, Parabolic singular integrals of Calderón-type, rough operators, and caloric layer potentials, Duke Math. J. 90 (1997), no. 2, 209-259, DOI 10.1215/S0012-7094-97-090086. MR:1484857(99b:42018) 
[7] Steve Hofmann and John L. Lewis, Square functions of Calderón type and applications, Rev. Mat. Iberoamericana 17 (2001), no. 1, 1-20, DOI 10.4171/RMI/287. MR 1846089 (2002g:47067)

[8] Steve Hofmann, Dorina Mitrea, Marius Mitrea, and Andrew J. Morris, $L^{p}$-square function estimates on spaces of homogeneous type and on uniformly rectifiable sets, Mem. Amer. Math. Soc., to appear, available at http://arxiv.org/abs/1301.4943.

[9] Peter W. Jones, Square functions, Cauchy integrals, analytic capacity, and harmonic measure, Harmonic analysis and partial differential equations (El Escorial, 1987), Lecture Notes in Math., vol. 1384, Springer, Berlin, 1989, pp. 24-68, DOI 10.1007/BFb0086793. MR 1013815 (91b:42032)

[10] Xavier Tolsa, Uniform rectifiability, Calderón-Zygmund operators with odd kernel, and quasiorthogonality, Proc. Lond. Math. Soc. (3) 98 (2009), no. 2, 393-426, DOI 10.1112/plms/pdn035. MR2481953 (2010f:28007)

Department of Mathematics and Statistics, University of Helsinki, P.O.B. 68, FI00014 Helsinki, Finland

E-mail address: henri.martikainen@helsinki.fi

Departament de Matemàtiques, Universitat Autònoma de Barcelona and Centre de Reserca Matemàtica, Edifici C Facultat de Ciències, 08193 Bellaterra, Barcelona, SPAIN

E-mail address: mmourgoglou@crm.cat 